\title{
Drivers of Restoration Trajectory of a Community of Regenerant Plants: Natural Regeneration or Tree Seedling?
}

\author{
Emerson Viveiros ${ }^{1}$ \\ Bruno Santos Francisco ${ }^{1}$ \\ Alex Mauri Tello López ${ }^{1}$ \\ Fatima Conceição Márquez Piña-Rodrigues ${ }^{1,2}$ \\ José Mauro Santana da Silva ${ }^{1,2}$
}

${ }^{1}$ Universidade Federal de São Carlos, Programa de Pós-graduação em Planejamento e Uso de Recursos Renováveis, Sorocaba, SP, Brasil.

${ }^{2}$ Universidade Federal de São Carlos, Departamento de Ciências Ambientais, Sorocaba, SP, Brasil.

\begin{abstract}
Tree plantation is the most used technique for ecological restoration, although passive restoration requires a lower investment of resources. This research aimed to compare the composition and structure of the community of regenerant plants in areas under restoration through Assisted Natural Regeneration and mixed native Tree Planting, in the same area. We randomly placed 12 plots of $100 \mathrm{~m}^{2}$ each and sampled all the regenerating individuals found within them. We compared both restoration methods regarding growth pattern, dispersal syndromes, and successional groups. In both methodologies, trees and shrubs were the most abundant growth forms. The pioneer successional class was the most abundant in number of individuals for both treatments. We concluded that the two restoration methods allowed the regeneration of native species. We propose that Assisted Natural Regeneration is a viable restoration strategy, capable of boosting the initial processes of the community, especially in a matrix surrounded by remaining forests.
\end{abstract}

Keywords: Seasonal forest, biodiversity, growth form, successional group, dispersal syndrome.

\section{INTRODUCTION}

Tree planting is the most used technique for ecological restoration (Schorn et al., 2010), although passive restoration requires a lower investment, especially in areas of difficult access (Leal-Filho et al., 2013), relying only on successional processes to recover spontaneously, like the arrival of propagules and seed banks (Chazdon, 2012). In this method, natural regeneration occurs in tropical forests through seeds and seedling soil banks, seed rain, and resprouters (Calegari et al., 2013; Piña-Rodrigues \& Aoki, 2014; Almeida, 2016).

The techniques of forest restoration aim to potentially maximize the environmental resilience, favoring the natural mechanisms that result in the natural recovery of the environment (Campello, 1998). In general, they must recreate ecologically viable communities by protecting and fostering the natural ability of ecosystems to change along the process of ecological succession (Oliveira \& Engel, 2018). However, the success of restoration through Assisted Natural Regeneration is linked to the characteristics of the surrounding landscape (Pereira et al., 2013), the conditions of the site, the control and management of invasive grasses (Souza et al., 2012; Freitas et al., 2019; Rodrigues et al., 2019), and species functional traits (Passareti et al., 2020). In the tropics, natural regeneration creates mosaics of fragments of different ages and history of disturbances (Schilling et al., 2016), making secondary forests a common forest type on the landscape. Forest remnants vary in composition and biotic and abiotic characteristics, which affect the supply of seeds to its surroundings (Santos, 2018).

Following the actual global climate change scenario, Brazil will reach the highest expected losses in forest vegetation cover by 2050 (Bastin et al., 2019). In Brazil, there is currently a liability of approximately 21 million hectares of protected areas that must be restored (Sae, 2013; Soares Filho et al., 2014; Planaveg, 2017). International commitments undertaken by Brazil involve the restoration of 12.5 million hectares by 2030 , of which 2.2 million hectares represent passive restoration through natural regeneration (Brasil, 2017). 
The development of low-cost restoration models contributes to the rapid expansion of forest regeneration. Given the potential of restoration through natural recovery, it is critical to analyze the development of ecological processes that enable forests under restoration to reach the desired scenario which, in this case, is the reestablishment of the original forest cover.

Our hypothesis is that the ecological restoration by natural regeneration is as efficient as restoration by conventional planting of seedlings, as long as there is adequate management on the area, such as control of leaf-cutting ants and exotic grasses. Furthermore, we believe that nearby forest remnants contribute to the arrival of propagules for the succession of the areas. For this purpose, we have compared, on the same landscape, the composition and structure of regenerating communities in areas restored by Assisted Natural Regeneration and mixed native Tree Planting.

\section{MATERIAL AND METHODS}

\subsection{Study site}

This study was carried out in 4.73 hectares under restoration in the municipality of Borborema, São Paulo State, Brazil (21 ${ }^{\circ} 43^{\prime} 38.94^{\prime \prime}$ ' latitude, $49^{\circ} 3^{\prime} 1.18^{\prime \prime}$ W longitude) (Figure 1). The altitude varies from 384 to $387 \mathrm{~m}$ asl, and the vegetation is represented by remnants of the tropical seasonal semideciduous forest. The climate is tropical, with dry winters, classified as $A w$ by Köeppen (1948). The annual mean temperature and rainfall are $22.2^{\circ} \mathrm{C}$ and $1,231 \mathrm{~mm}$, respectively. The driest month is August, with a historical average rainfall of $19 \mathrm{~mm}$. The warmest and wettest month is January, with a mean precipitation of $234 \mathrm{~mm}$ and a temperature of $24.8^{\circ} \mathrm{C}$. The average temperature in June, the coldest month, is $18.5^{\circ} \mathrm{C}$ (Climate-Data, 2019). The area is located in a zone seized by the "Promissão" hydroelectric dam, in which the fodder grass Paspalum notatum Flüggé is used as pasture. In order to run the experiment, the expropriation elevation level of the reservoir (i.e., the area buffering the water reservoir where agricultural and construction activities are prohibited for safety reasons), as well as the normal maximum elevation level were identified through georeferencing equipment.

Adjacent to the study area, a fragment of approximately 4 hectares of tropical seasonal semideciduous forest with secondary vegetation, in an intermediary stage of regeneration, presents tree and shrub plants covering the herbaceous vegetation. The trees have approximately 10 meters high, with some higher individuals. The canopy is discontinuous and has openings with a predominance of shrub and herbaceous species. We have observed epiphytes and the presence of mainly woody vines, and litter distributed throughout the entire area. We observed some species such as Trema micrantha (L.) Blume, Croton floribundus Spreng., Albizia niopoides (Benth.) Burkart, Schinus terebinthifolia Raddi, Aloysia virgata (Ruiz \& Pav.) Juss., Rhamnidium elaeocarpum Reissek, Vitex megapotamica (Spreng.) Moldenke, Mabea fistulifera Mart., Solanum paniculatum L., Ananas sp., and Psychotria carthagenensis Jacq.

\subsubsection{Restoration methods}

In October 2014, the study area was fenced to reduce external degradation factors such as cattle grazing and others. After that, we have implemented two restoration methods: (a) Assisted Natural Regeneration (ANR) and (b) mixed native seedlings Tree Planting (TP). The ANR occupied 2.47 ha and the TP 2.26 ha. In November 2014, we carried out the suppression of leaf-cutter ants and the application of 2.5 liters.ha ${ }^{-1}$ of glyphosate ( $\mathrm{N}$-phosphonomethyl glycine) in both areas for the control of the non-native invasive grass P. notatum. Another round of the herbicide was applied again every semester for two years in the ANR.

Fifteen days after the first suppression of invasive grasses in TP, in November 2014, 1 ton.ha ${ }^{-1}$ of dolomitic limestone was applied, followed by harrowing. We subsoiled the harrowed area to $60 \mathrm{~cm}$ depth, with a 3-meter spacing between the subsoiling lines and applied a chemical N:P:K (20:5:20; 660 kg.ha $\left.{ }^{-1}\right)$ superphosphate fertilizer. In December 2014, seedlings from 97 native species were planted using manual planters, with a. spacing of $3 \times 2 \mathrm{~m}$ (Additional file 1). Later, we carried out the maintenance activities every four months during three years, controlling the invasive grasses with herbicides ( 2.5 liters.ha $^{-1}$ of glyphosate), seedling fertilization with N:P:K (20:5:20; $400 \mathrm{~kg} \cdot \mathrm{ha}^{-1}$ ), seedlings crowning, and leaf-cutting ants control.

In both experimental areas the soil type was the same, being characterized as deep friable soils, with high drainage. The main types of soil found in the area are red latosols and argisols, with basalt rocks being found in the region.

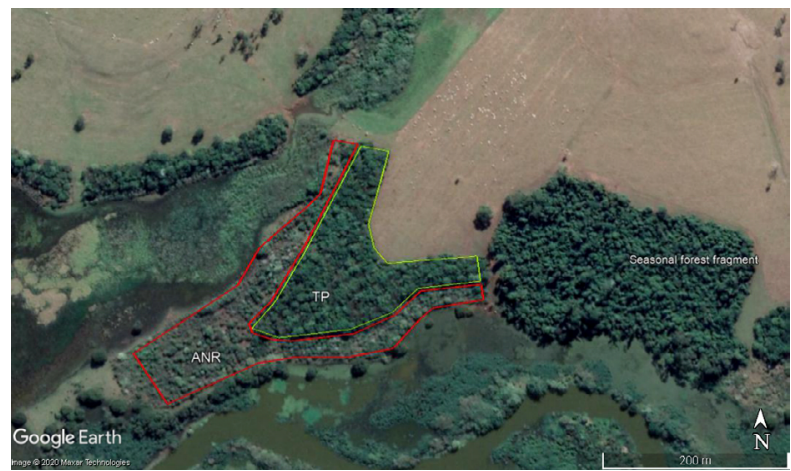

Figure 1. Location of the study areas around the hydroelectric reservoir in Borborema, São Paulo, Brazil. ANR = Assisted Natural Regeneration, $\mathrm{TP}=$ mixed native Tree Planting. 


\subsection{Data sample}

\subsubsection{Inventory and functional classification}

We have randomly placed six 4 × $25 \mathrm{~m}$ permanent plots after four and three years for TP and ANR, respectively, totaling 12 permanent plots of $100 \mathrm{~m}^{2}$ each. All individuals higher than $50 \mathrm{~cm}$ high and with a circumference at breast height $(\mathrm{CBH})$ lower than $15 \mathrm{~cm}$ were classified as regenerants, and were measured and identified to the lowest possible taxonomic level. In the TP, we have considered regenerants as the nonplanted individuals mainly found between planting rows.

The species were identified in the field, or later, in the herbarium, when necessary. The spelling of the scientific names, the valid names, and the synonyms were checked according to The Plant List (2013). In order to determine different functional groups, all species were classified according to their growth form, successional group, and dispersal syndrome according to the São Paulo database of species for restoration (Barbosa et al., 2017).

\subsection{Data Analyses}

We have calculated the phytosociological parameters for shrubs and trees according to Moro \& Martins (2011). The species diversity was calculated using the ShannonWeaver Index ( $\left.\mathrm{H}^{\prime}\right)$ and the Equitability was calculated based on Pielou (J'). The species distribution in the community was evaluated by the Sorensen similarity coefficient (Martins \& Santos, 1999), in order to compare the floristic similarity between the restoration methods and the list of species planted in TP.

The abundance and richness of regenerants were calculated and the restoration methods were compared using the Student's $\mathrm{t}$-test. We compared the percentage of growth forms, dispersal syndromes, and successional groups between the restoration methods through the Kruskal-Wallis non-parametric test (both at $5 \%$ probability level). When significant differences were detected, Dunn's tests were performed. All statistical analyses were carried out using the R software (R Core Team, 2020).

\section{RESULTS}

We sampled a total of 981 individuals (estimated 8,175 individuals.ha $^{-1}$ ), distributed in 66 species and 24 botanical families (Table 1). Only six species totaled $67.8 \%$ of the individuals, namely Senegalia polyphylla (184 individuals), Vernonanthura polyanthes (142), Sida santaremensis (133), Solanum paniculatum (106), Solanum mauritianum (58), and Desmodium incanum (42). The botanical families that accounted for $55.5 \%$ of species richness and that represented $85.1 \%$ of the density were the following: Asteraceae (10 species), Malvaceae (nine), Fabaceae (six), Solanaceae (five), Myrtaceae (four), and Verbenaceae (four). Four herbaceous species in the ANR were not identified.

Table 1. List of regenerant species ordered by family and sampled in restoration sites at the region of Borborema, São Paulo State, Brazil, containing the abundance (number of individuals), growth form, dispersal syndrome, and successional group, for different restoration methods. ANR: Assisted Natural Regeneration, TP: Tree Planting; Anemo: wind dispersal; Zoo: animal dispersal; Auto: another dispersal strategy; P: pioneer; NP: non-pioneer.

\begin{tabular}{|c|c|c|c|c|c|c|}
\hline \multirow{2}{*}{ Species } & \multirow{2}{*}{ Family } & \multicolumn{2}{|c|}{ Abundance } & \multirow{2}{*}{ Growth Form } & \multirow{2}{*}{$\begin{array}{l}\text { Dispersal } \\
\text { Syndrome }\end{array}$} & \multirow{2}{*}{$\begin{array}{l}\text { Successional } \\
\text { Group }\end{array}$} \\
\hline & & ANR & TP & & & \\
\hline Astronium graveolens Jacq. & Anacardiaceae & 0 & 2 & Tree & Anemo & NP \\
\hline $\begin{array}{l}\text { Schinus terebinthifolia } \\
\text { Raddi }\end{array}$ & Anacardiaceae & 3 & 3 & Tree & Zoo & $\mathrm{P}$ \\
\hline Tapirira guianensis Aubl. & Anacardiaceae & 0 & 2 & Tree & Zoo & NP \\
\hline $\begin{array}{l}\text { Oxypetalum } \\
\text { appendiculatum Mart. }\end{array}$ & Apocynaceae & 0 & 5 & Vine & Anemo & $\mathrm{P}$ \\
\hline $\begin{array}{l}\text { Acrocomia aculeata (Jacq.) } \\
\text { Lodd. ex Mart. }\end{array}$ & Arecaceae & 5 & 3 & Tree & Zoo & NP \\
\hline Asteraceae 1 & Asteraceae & 0 & 1 & Herb & Anemo & $\mathrm{P}$ \\
\hline $\begin{array}{l}\text { Chromolaena maximiliani } \\
\text { (Schrad. ex DC.) R.M. } \\
\text { King \& H. Rob. }\end{array}$ & Asteraceae & 17 & 0 & Shrub & Anemo & $\mathrm{P}$ \\
\hline $\begin{array}{l}\text { Emilia sonchifolia (L.) DC. } \\
\text { ex Wight }\end{array}$ & Asteraceae & 1 & 0 & Herb & Anemo & $\mathrm{P}$ \\
\hline $\begin{array}{l}\text { Erechtites hieracifolius (L.) } \\
\text { Raf. ex DC. }\end{array}$ & Asteraceae & 7 & 0 & Herb & Anemo & $\mathrm{P}$ \\
\hline Galinsoga parviflora Cav. & Asteraceae & 1 & 0 & Herb & Anemo & $\mathrm{P}$ \\
\hline
\end{tabular}


Table 1. Continued..

\begin{tabular}{|c|c|c|c|c|c|c|}
\hline \multirow{2}{*}{ Species } & \multirow{2}{*}{ Family } & \multicolumn{2}{|c|}{ Abundance } & \multirow{2}{*}{ Growth Form } & \multirow{2}{*}{$\begin{array}{l}\text { Dispersal } \\
\text { Syndrome }\end{array}$} & \multirow{2}{*}{$\begin{array}{c}\text { Successiona } \\
\text { Group }\end{array}$} \\
\hline & & ANR & TP & & & \\
\hline $\begin{array}{l}\text { Mikania campanulata } \\
\text { Gardner }\end{array}$ & Asteraceae & 0 & 2 & Vine & Anemo & $\mathrm{P}$ \\
\hline $\begin{array}{l}\text { Porophyllum ruderale } \\
\text { (Jacq.) Cass. }\end{array}$ & Asteraceae & 5 & 0 & Herb & Anemo & $\mathrm{P}$ \\
\hline $\begin{array}{l}\text { Pterocaulon lanatum } \\
\text { Kuntze }\end{array}$ & Asteraceae & 8 & 1 & Shrub & Anemo & $\mathrm{P}$ \\
\hline Solidago chilensis Meyen & Asteraceae & 1 & 0 & Shrub & Anemo & $\mathrm{P}$ \\
\hline $\begin{array}{l}\text { Vernonanthura polyanthes } \\
\text { (Sprengel) Vega \& } \\
\text { Dematteis }\end{array}$ & Asteraceae & 134 & 8 & Shrub & Anemo & $\mathrm{P}$ \\
\hline $\begin{array}{l}\text { Handroanthus ochraceus } \\
\text { (Cham.) Mattos }\end{array}$ & Bignoniaceae & 0 & 3 & Tree & Anemo & NP \\
\hline $\begin{array}{l}\text { Trema micrantha } \\
\text { (L.) Blume }\end{array}$ & Cannabaceae & 1 & 2 & Tree & Zoo & $\mathrm{P}$ \\
\hline Ipomoea nil (L.) Roth & Convolvulaceae & 0 & 1 & Vine & Auto & $\mathrm{P}$ \\
\hline $\begin{array}{l}\text { Erythroxylum pelleterianum } \\
\text { A.St.-Hil. }\end{array}$ & Erythroxylaceae & 0 & 6 & Shrub & Zoo & $\mathrm{NP}$ \\
\hline Croton floribundus Spreng. & Euphorbiaceae & 0 & 3 & Tree & Auto & $\mathrm{P}$ \\
\hline Mabea fistulifera Mart. & Euphorbiaceae & 14 & 7 & Tree & Auto & $\mathrm{P}$ \\
\hline $\begin{array}{l}\text { Albizia niopoides (Benth.) } \\
\text { Burkart }\end{array}$ & Fabaceae & 1 & 0 & Tree & Auto & $\mathrm{P}$ \\
\hline Desmodium incanum DC. & Fabaceae & 36 & 6 & Shrub & Zoo & $\mathrm{P}$ \\
\hline $\begin{array}{l}\text { Enterolobium } \\
\text { contortisiliquum } \\
\text { (Vell.) Morong }\end{array}$ & Fabaceae & 1 & 0 & Tree & Auto & $\mathrm{P}$ \\
\hline $\begin{array}{l}\text { Parapiptadenia rigida } \\
\text { (Benth.) Brenan }\end{array}$ & Fabaceae & 1 & 0 & Tree & Auto & $\mathrm{NP}$ \\
\hline $\begin{array}{l}\text { Peltophorum dubium } \\
\text { (Spreng.) Taub. }\end{array}$ & Fabaceae & 0 & 1 & Tree & Auto & $\mathrm{P}$ \\
\hline $\begin{array}{l}\text { Senegalia polyphylla (DC.) } \\
\text { Britton \& Rose }\end{array}$ & Fabaceae & 8 & 176 & Tree & Auto & $\mathrm{P}$ \\
\hline $\begin{array}{l}\text { Aegiphila sellowiana } \\
\text { Cham. }\end{array}$ & Lamiaceae & 0 & 1 & Tree & Zoo & $\mathrm{P}$ \\
\hline $\begin{array}{l}\text { Hyptis suaveolens } \\
\text { (L.) Poit. }\end{array}$ & Lamiaceae & 0 & 1 & Herb & Auto & $\mathrm{P}$ \\
\hline $\begin{array}{l}\text { Ocimum campechianum } \\
\text { Mill. }\end{array}$ & Lamiaceae & 1 & 0 & Herb & Auto & $\mathrm{P}$ \\
\hline $\begin{array}{l}\text { Nectandra lanceolata } \\
\text { Nees \& Mart. }\end{array}$ & Lauraceae & 2 & 2 & Tree & Zoo & $\mathrm{NP}$ \\
\hline $\begin{array}{l}\text { Guazuma } \\
\text { ulmifolia Lam. }\end{array}$ & Malvaceae & 1 & 1 & Tree & Zoo & $\mathrm{P}$ \\
\hline Malvaceae 1 & Malvaceae & 0 & 4 & Shrub & - & - \\
\hline Sida cordifolia L. & Malvaceae & 2 & 0 & Shrub & Auto & $\mathrm{P}$ \\
\hline Sida rhombifolia L. & Malvaceae & 7 & 3 & Shrub & Auto & $\mathrm{P}$ \\
\hline $\begin{array}{l}\text { Sida santaremensis } \\
\text { Monteiro }\end{array}$ & Malvaceae & 97 & 36 & Shrub & Auto & $\mathrm{P}$ \\
\hline $\begin{array}{l}\text { Sidastrum paniculatum } \\
\text { (L.) Fryxell }\end{array}$ & Malvaceae & 15 & 0 & Shrub & Auto & $\mathrm{P}$ \\
\hline Urena lobata L. & Malvaceae & 5 & 3 & Shrub & Zoo & $\mathrm{P}$ \\
\hline $\begin{array}{l}\text { Waltheria communis } \\
\text { A.St.-Hil. }\end{array}$ & Malvaceae & 1 & 0 & Shrub & Auto & $\mathrm{P}$ \\
\hline
\end{tabular}

Continues... 
Table 1. Continued...

\begin{tabular}{|c|c|c|c|c|c|c|}
\hline \multirow{2}{*}{ Species } & \multirow{2}{*}{ Family } & \multicolumn{2}{|c|}{ Abundance } & \multirow{2}{*}{ Growth Form } & \multirow{2}{*}{$\begin{array}{l}\text { Dispersal } \\
\text { Syndrome }\end{array}$} & \multirow{2}{*}{$\begin{array}{l}\text { Successional } \\
\text { Group }\end{array}$} \\
\hline & & ANR & $\mathrm{TP}$ & & & \\
\hline $\begin{array}{l}\text { Wissadula subpeltata } \\
\text { (Kuntze) R.E.Fr. }\end{array}$ & Malvaceae & 1 & 11 & Shrub & Auto & $\mathrm{NP}$ \\
\hline $\begin{array}{l}\text { Guarea guidonia (L.) } \\
\text { Sleumer }\end{array}$ & Meliaceae & 5 & 0 & Tree & Zoo & NP \\
\hline Trichilia pallida Sw. & Meliaceae & 1 & 2 & Tree & Zoo & NP \\
\hline Myrtaceae 1 & Myrtaceae & 0 & 7 & Tree & Zoo & $\mathrm{NP}$ \\
\hline $\begin{array}{l}\text { Psidium cattleianum } \\
\text { Sabine }\end{array}$ & Myrtaceae & 1 & 0 & Tree & Zoo & NP \\
\hline Psidium guajava L. & Myrtaceae & 22 & 0 & Tree & Zoo & $\mathrm{P}$ \\
\hline $\begin{array}{l}\text { Syzygium cumini (L.) } \\
\text { Skeels }\end{array}$ & Myrtaceae & 5 & 0 & Tree & Zoo & $\mathrm{P}$ \\
\hline Passiflora edulis Sims & Passifloraceae & 0 & 1 & Vine & Zoo & $\mathrm{P}$ \\
\hline Myrsine umbellata Mart. & Primulaceae & 0 & 1 & Tree & Zoo & NP \\
\hline $\begin{array}{l}\text { Rhamnidium elaeocarpum } \\
\text { Reissek }\end{array}$ & Rhamnaceae & 2 & 4 & Tree & Zoo & $\mathrm{NP}$ \\
\hline $\begin{array}{l}\text { Psychotria carthagenensis } \\
\text { Jacq. }\end{array}$ & Rubiaceae & 8 & 1 & Shrub & Zoo & NP \\
\hline Serjania sp. & Sapindaceae & 1 & 0 & Vine & Anemo & NP \\
\hline Smilax campestris Griseb. & Smilacaceae & 21 & 10 & Vine & Zoo & $\mathrm{P}$ \\
\hline Solanum americanum Mill. & Solanaceae & 6 & 0 & Shrub & Zoo & $\mathrm{P}$ \\
\hline Solanum capsicoides All. & Solanaceae & 8 & 0 & Shrub & Zoo & $\mathrm{P}$ \\
\hline $\begin{array}{l}\text { Solanum mauritianum } \\
\text { Scop. }\end{array}$ & Solanaceae & 58 & 0 & Tree & Zoo & NP \\
\hline $\begin{array}{l}\text { Solanum palinacanthum } \\
\text { Dunal }\end{array}$ & Solanaceae & 3 & 0 & Shrub & Zoo & $\mathrm{P}$ \\
\hline Solanum paniculatum L. & Solanaceae & 73 & 33 & Shrub & Zoo & $\mathrm{P}$ \\
\hline Unknown 1 & Unknown 1 & 1 & 0 & Herb & - & - \\
\hline Unknown 2 & Unknown 2 & 1 & 0 & Herb & - & - \\
\hline Unknown 3 & Unknown 3 & 6 & 0 & Herb & - & - \\
\hline Unknown 4 & Unknown 4 & 11 & 0 & Herb & - & - \\
\hline $\begin{array}{l}\text { Cecropia } \\
\text { pachystachyaTrécul }\end{array}$ & Urticaceae & 2 & 1 & Tree & Zoo & $\mathrm{P}$ \\
\hline $\begin{array}{l}\text { Aloysia virgata (Ruiz \& } \\
\text { Pav.) Juss. }\end{array}$ & Verbenaceae & 0 & 10 & Tree & Auto & $\mathrm{P}$ \\
\hline $\begin{array}{l}\text { Citharexylum myrianthum } \\
\text { Cham. }\end{array}$ & Verbenaceae & 1 & 0 & Tree & Zoo & $\mathrm{P}$ \\
\hline Lantana camara L. & Verbenaceae & 0 & 4 & Shrub & Zoo & $\mathrm{P}$ \\
\hline $\begin{array}{l}\text { Stachytarpheta cayennensis } \\
\text { (Rich.) Vahl }\end{array}$ & Verbenaceae & 1 & 0 & Shrub & Auto & $\mathrm{P}$ \\
\hline TOTAL & & 613 & 368 & & & \\
\hline
\end{tabular}


The species with the highest IVI in the ANR treatment were $S$. mauritianum (IVI=67.54; 22.5\%), $S$. paniculatum (56.96; 18.9\%), Psidium guajava (51.26; 17\%), Mabea fistulifera (20.12; 6.7\%), and Acrocomia aculeata (12.56; 4.2\%), which together represented $78.9 \%$ of the sampled individuals and $69.5 \%$ of the IVI (Table 2), with dominance of the Solanaceae family (58.1\% of the individuals).
The species with highest IVI in the TP treatment were S. polyphylla $(\mathrm{IVI}=94.08 ; 31.4 \%)$; . paniculatum $(39.79 ; 13.3 \%)$; Handroanthus ochraceus (18.94; 6.3\%); Aloysia virgata (18.70; $6.2 \%)$; and Schinus terebinthifolia $(\mathrm{IVI}=17.30 ; 5.8 \%)$, which represented $83 \%$ of the individuals and $63 \%$ of the IVI for this restoration method. Fabaceae and Solanaceae showed the highest dominance (Table 3 ).

Table 2. Phytosociology of regenerant species (trees and shrubs) sampled in the Assisted Natural Regeneration (ANR) area, at the region of Borborema, São Paulo State, Brazil. N = number of individuals sampled; $\mathrm{AD}=$ absolute density; $\mathrm{RD}=$ relative density; $\mathrm{AF}=\mathrm{absolute}$ frequency; $\mathrm{RF}=$ relative frequency; $\mathrm{ADo}=$ absolute dominance; $\mathrm{RDo}=$ relative dominance; $\mathrm{CVI}=$ Crown Value Index; $\mathrm{IVI}=\mathrm{Importance}$ Value Index.

\begin{tabular}{|c|c|c|c|c|c|c|c|c|c|}
\hline Species & $\mathbf{N}$ & AD & RD & AF & RF & ADo & RDo & CVI & IVI \\
\hline Solanum mauritianum & 58 & 966.7 & 26.61 & 100 & 12.5 & 1.65 & 28.43 & 55.04 & 67.54 \\
\hline Solanum paniculatum & 73 & 1216.7 & 33.49 & 100 & 12.5 & 0.64 & 10.97 & 44.46 & 56.96 \\
\hline Psidium guajava & 22 & 366.7 & 10.09 & 100 & 12.5 & 1.67 & 28.67 & 38.76 & 51.26 \\
\hline Mabea fistulifera & 14 & 233.3 & 6.42 & 33 & 4.17 & 0.55 & 9.54 & 15.96 & 20.12 \\
\hline Acrocomia aculeata & 5 & 83.3 & 2.29 & 67 & 8.33 & 0.11 & 1.93 & 4.23 & 12.56 \\
\hline Senegalia polyphylla & 8 & 133.3 & 3.67 & 50 & 6.25 & 0.12 & 02.01 & 5.68 & 11.93 \\
\hline Cecropia pachystachya & 2 & 33.3 & 0.92 & 33 & 4.17 & 0.28 & 4.81 & 5.72 & 9.89 \\
\hline Schinus terebinthifolia & 3 & 50.0 & 1.38 & 50 & 6.25 & 0.02 & 0.34 & 1.71 & 7.96 \\
\hline Syzygium cumini & 5 & 83.3 & 2.29 & 33 & 4.17 & 0.07 & 1.14 & 3.43 & 7.60 \\
\hline Enterolobium contortisiliquum & 1 & 16.7 & 0.46 & 17 & 02.08 & 0.26 & 4.42 & 4.88 & 6.96 \\
\hline Unknown & 3 & 50.0 & 1.38 & 33 & 4.17 & 0.04 & 0.63 & 02.01 & 6.17 \\
\hline Psychotria carthagenensis & 8 & 133.3 & 3.67 & 17 & 02.08 & 0.02 & 0.36 & 04.03 & 6.11 \\
\hline Citharexylum myrianthum & 1 & 16.7 & 0.46 & 17 & 02.08 & 0.20 & 3.47 & 3.93 & 6.01 \\
\hline Guarea guidonia & 5 & 83.3 & 2.29 & 17 & 02.08 & 0.06 & 1.11 & 3.40 & 5.48 \\
\hline Guazuma ulmifolia & 1 & 16.7 & 0.46 & 17 & 02.08 & 0.05 & 0.91 & 1.37 & 3.45 \\
\hline Nectandra lanceolata & 2 & 33.3 & 0.92 & 17 & 02.08 & 0.02 & 0.36 & 1.28 & 3.36 \\
\hline Rhamnidium elaeocarpum & 2 & 33.3 & 0.92 & 17 & 02.08 & 0.01 & 0.15 & 01.07 & 3.15 \\
\hline Parapiptadenia rigida & 1 & 16.7 & 0.46 & 17 & 02.08 & 0.02 & 0.39 & 0.84 & 2.93 \\
\hline Psidium cattleianum & 1 & 16.7 & 0.46 & 17 & 02.08 & 0.01 & 0.18 & 0.64 & 2.73 \\
\hline Trichilia pallida & 1 & 16.7 & 0.46 & 17 & 02.08 & 0.00 & 0.08 & 0.54 & 2.62 \\
\hline Trema micrantha & 1 & 16.7 & 0.46 & 17 & 02.08 & 0.00 & 0.06 & 0.52 & 2.60 \\
\hline Albizia niopoides & 1 & 16.7 & 0.46 & 17 & 02.08 & 0.00 & 0.04 & 0.50 & 2.58 \\
\hline
\end{tabular}

Table 3. Species (trees and shrubs) regenerating in the mixed native Tree Planting (TP), at the region of Borborema, São Paulo State, Brazil. Legend: $\mathrm{N}=$ number of individuals sampled; $\mathrm{AD}=$ absolute density; $\mathrm{RD}=$ relative density; $\mathrm{AF}=$ absolute frequency; $\mathrm{RF}=$ relative frequency; $\mathrm{ADo}=$ absolute dominance; RDo = relative dominance; IVI = Importance Value Index; and CVI = Crown Value Index.

\begin{tabular}{lcccccccccc}
\multicolumn{1}{c}{ Species } & N & AD & RD & AF & RF & ADo & RDo & CVI & IVI \\
Senegalia polyphylla & 176 & 2933.3 & 64.9 & 83 & 10.87 & 0.07 & 18.26 & 83.21 & 94.08 \\
\hline Solanum paniculatum & 33 & 550 & 12.2 & 50 & 6.52 & 0.08 & 21.09 & 33.27 & 39.79 \\
Handroanthus ochraceus & 3 & 50 & 1.1 & 33 & 4.35 & 0.05 & 13.48 & 14.59 & 18.94 \\
Aloysia virgata & 10 & 166.7 & 3.7 & 83 & 10.87 & 0.02 & 4.14 & 7.83 & 18.7 \\
Schinus terebinthifolia & 3 & 50 & 1.1 & 33 & 4.35 & 0.04 & 11.85 & 12.95 & 17.3
\end{tabular}


Table 3. Continued...

\begin{tabular}{|lccccccccc}
\multicolumn{1}{c}{ Species } & N & AD & RD & AF & RF & ADo & RDo & CVI & IVI \\
Mabea fistulifera & 7 & 116.7 & 2.6 & 50 & 6.52 & 0.02 & 4.15 & 6.73 & 13.26 \\
\hline Croton floribundus & 3 & 50 & 1.1 & 50 & 6.52 & 0.01 & 4.01 & 5.11 & 11.64 \\
Acrocomia aculeata & 3 & 50 & 1.1 & 50 & 6.52 & 0.01 & 2.07 & 3.18 & 9.7 \\
\hline Myrtaceae 1 & 7 & 116.7 & 2.6 & 33 & 4.35 & 0.01 & 1.85 & 4.44 & 8.78 \\
\hline Trema micrantha & 2 & 33.3 & 0.7 & 33 & 4.35 & 0.01 & 3.23 & 3.97 & 8.32 \\
\hline Rhamnidium elaeocarpum & 4 & 66.7 & 1.5 & 33 & 4.35 & 0.01 & 1.82 & 3.29 & 7.64 \\
\hline Tapirira guianensis & 2 & 33.3 & 0.7 & 33 & 4.35 & 0.01 & 1.89 & 2.63 & 6.98 \\
\hline Guazuma ulmifolia & 1 & 16.7 & 0.4 & 17 & 2.17 & 0.02 & 4.4 & 4.77 & 6.94 \\
\hline Erythroxylum pelleterianum & 6 & 100 & 2.2 & 17 & 2.17 & 0.01 & 1.93 & 4.14 & 6.31 \\
\hline Trichilia pallida & 2 & 33.3 & 0.7 & 33 & 4.35 & 0 & 0.91 & 1.65 & 5.99 \\
Nectandra lanceolata & 2 & 33.3 & 0.7 & 33 & 4.35 & 0 & 0.26 & 1 & 5.35 \\
\hline Myrsine umbellata & 1 & 16.7 & 0.4 & 17 & 2.17 & 0 & 1.31 & 1.68 & 3.85 \\
\hline Astronium graveolens & 2 & 33.3 & 0.7 & 17 & 2.17 & 0 & 0.91 & 1.65 & 3.82 \\
\hline Cecropia pachystachya & 1 & 16.7 & 0.4 & 17 & 2.17 & 0 & 0.91 & 1.28 & 3.45 \\
\hline Aegiphila sellowiana & 1 & 16.7 & 0.4 & 17 & 2.17 & 0 & 0.91 & 1.28 & 3.45 \\
\hline Peltophorum dubium & 1 & 16.7 & 0.4 & 17 & 2.17 & 0 & 0.58 & 0.95 & 3.12 \\
\hline Psychotria carthagenensis & 1 & 16.7 & 0.4 & 17 & 2.17 & 0 & 0.04 & 0.41 & 2.58 \\
\hline
\end{tabular}

In both methods of restoration, A. aculeata, Cecropia pachystachya, Guazuma ulmifolia, M. fistulifera, Nectandra lanceolata, Psychotria carthagenensis, Rhamnidium elaeocarpum, S. terebinthifolia, S. polyphylla, and S. paniculatum were part of the community of regenerants, representing $37.5 \%$ of all the sampled species (32 species). Among the total number of regenerant species found in each method $(\mathrm{ANR}=218$; $\mathrm{TP}=271$ ), the common species represented 55\% (ANR) and 83.3\% (TP) of individuals, but they differed in abundance $(\mathrm{T}=2.38$, $\mathrm{p}$-value $=0.03)$.

Table 4. Parameters of structure, density, and richness of the community of regenerants in areas under restoration using the Assisted Natural Regeneration (ANR) and mixed native species Tree Planting (TP) methodologies, at the age of four years, at the Borborema region, state of São Paulo, Brazil. Implementation and planting were carried out in 2014.

\begin{tabular}{|c|c|c|c|}
\hline Parameters & Total & (ANR) & (TP) \\
\hline Abundance (n) & 981 & 613 & 368 \\
\hline Density (individuals.ha-1) & 8,175 & 10,216 & 6,133 \\
\hline Shannon-Weaver Index (H’) & & 2.61 & 2.26 \\
\hline Pielou Equability $\left(J^{\prime}\right)$ & & 0.682 & 0.622 \\
\hline Sorensen similarity index & & 0.48 & \\
\hline Sorensen similarity index for species planted in TP & & 0.08 & 0.09 \\
\hline Families & 24 & 18 & 23 \\
\hline Genera & 52 & 37 & 35 \\
\hline Species & 66 & 44 & 35 \\
\hline \multicolumn{4}{|l|}{ Growth form (richness) } \\
\hline Tree & & $19(45 \%)$ & $19(57 \%)$ \\
\hline Shrub & & $18(40 \%)$ & $12(34 \%)$ \\
\hline Herb & & $9(20 \%)$ & $2(0.5 \%)$ \\
\hline Vine & & $2(5 \%)$ & $5(14 \%)$ \\
\hline
\end{tabular}

Continues... 
Table 3. Continued...

\begin{tabular}{|c|c|c|}
\hline Parameters & (ANR) & $(\mathrm{TP})$ \\
\hline \multicolumn{3}{|l|}{ Successional group (richness) } \\
\hline Pioneers & $33(75 \%)$ & $25(71 \%)$ \\
\hline Non-pioneers & $11(25 \%)$ & $12(34 \%)$ \\
\hline \multicolumn{3}{|l|}{ Dispersal syndrome (richness) } \\
\hline Zoochoric & $22(50 \%)$ & $20(57 \%)$ \\
\hline Autochoric & $13(29 \%)$ & $10(28 \%)$ \\
\hline Anemochoric & $9(20 \%)$ & $7(20 \%)$ \\
\hline \multicolumn{3}{|l|}{ Growth form (abundance) } \\
\hline Tree & $134(33 \%)$ & $231(71 \%)$ \\
\hline Shrub & $423(69 \%)$ & $116(31 \%)$ \\
\hline Herb & $34(5 \%)$ & $2(0.5 \%)$ \\
\hline Vine & $22(3 \%)$ & $19(5 \%)$ \\
\hline \multicolumn{3}{|l|}{ Dispersal syndrome (abundance) } \\
\hline Zoochoric & $269(43 \%)$ & $93(25 \%)$ \\
\hline Autochoric & $150(24 \%)$ & $249(67 \%)$ \\
\hline Anemochoric & $175(28 \%)$ & $22(5 \%)$ \\
\hline \multicolumn{3}{|l|}{ Successional group (abundance) } \\
\hline Pioneers & $509(83 \%)$ & $320(86 \%)$ \\
\hline Non-pioneers & $85(13 \%)$ & $44(11 \%)$ \\
\hline
\end{tabular}

Although the ANR treatment had a significantly higher abundance of regenerants than the TP treatment (Table 4, Figure 2), the diversity and the equitability were similar, with a $48 \%$ similarity in the floristic composition between the two methodologies. In both restoration methods, trees were the dominant growth form (41\%) compared to shrubs (33\%) and other life forms (herbs $17 \%$ and vines $9 \%$ ), with the abundance of tree growth forms not being different from that of shrubs $(t=2.3816$, $\mathrm{p}$-value $=0.038$, although both were significantly different from the other growth forms (vines and herbs), in both treatments (Figure 3).

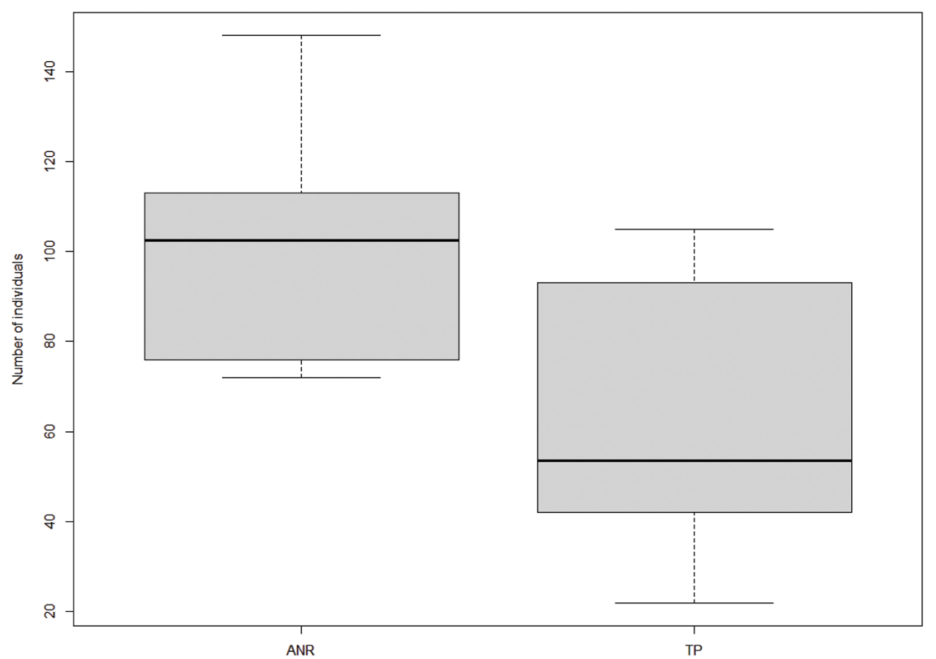

Figure 2. Boxplot of the abundance of regenerants in the two sampled areas, at the Borborema region, state of São Paulo, Brazil. Legend: $\mathrm{ANR}=$ number of regenerants in the Assisted Natural Regeneration area and TP = number of regenerants in the mixed native species Tree Planting (TP) area. 


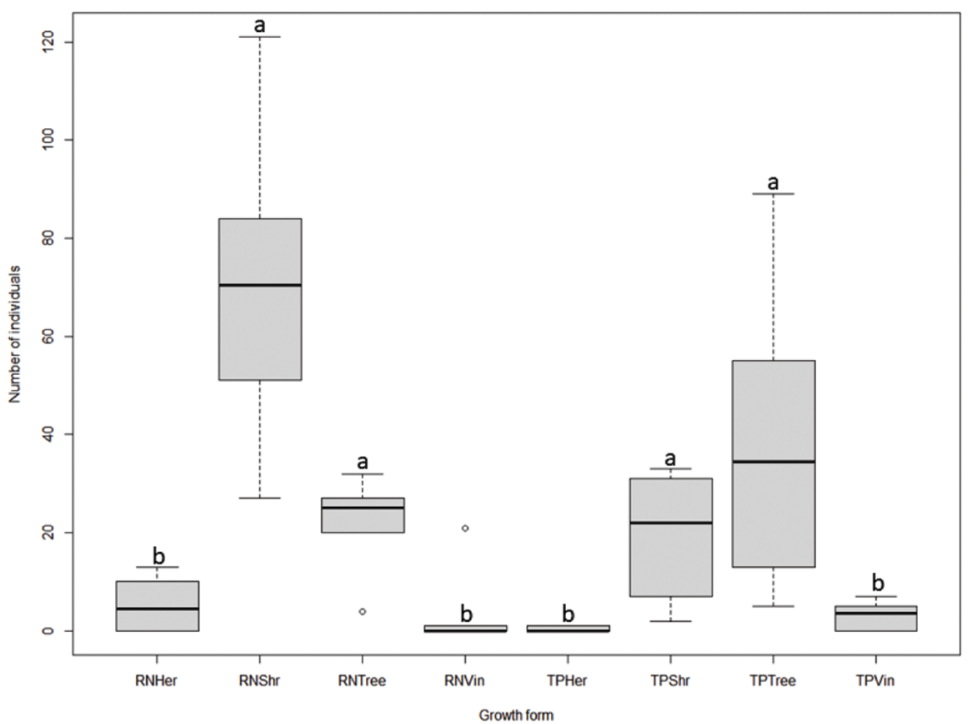

Figure 3. Boxplot of the abundance of regenerants per growth form in the Assisted Natural Regeneration (ANR) and mixed native Tree Planting (TP) methods, at the Borborema region, state of São Paulo, Brazil. Legend: RNtree = number of tree regenerants in the Natural Regeneration Area, RNshr = number of shrub regenerants in the Natural Regeneration Area, RNvin = number of vine regenerants in the Natural Regeneration Area, RNher = number of herbaceous regenerant in the Natural Regeneration Area, TPtree $=$ number of tree regenerants in the mixed native species Tree Planting Area, TPshr = number of shrub regenerants in the mixed native species Tree Planting Area, TPvin $=$ number of vine regenerants in the mixed native species Tree Planting Area, TPher $=$ number of herbaceous regenerant in the mixed native species Tree Planting Area. Letters above boxes indicates statistically significant differences.

The number of individuals with anemochoric and zoochoric dispersion syndromes was significantly different between the two regeneration methods $(H=17.05$, p-value $<0.01$ ), being more abundant in the RN (Figure 4). Whereas autochory dominated in the TP treatment, the syndromes did not differ in the ANR treatment. Within each method, the successional groups differed from each other $(\mathrm{H}=18.20$, $\mathrm{p}$-value $<0.01)$, with pioneers being more abundant in the ANR treatment than in the TP treatment (Figure 5).

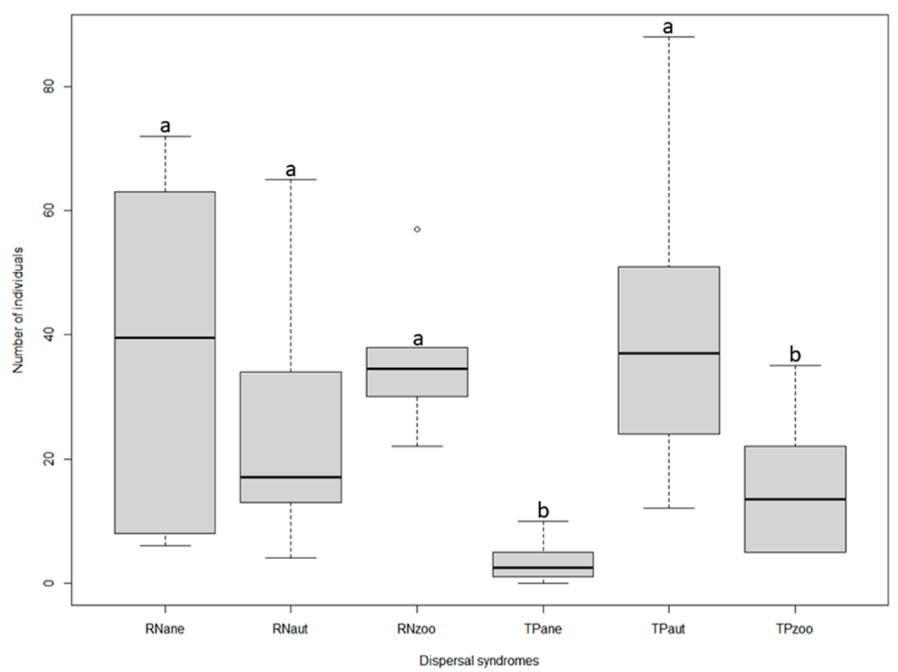

Figure 4. Boxplot of the abundance of regenerants according to their dispersal syndrome in the sampled areas (3A), at the Borborema region, state of São Paulo, Brazil. Legend: RNane = number of regenerants of anemochoric dispersal syndrome in the Natural Regeneration Area, $\mathrm{RNzoo}=$ number of regenerants with zoochoric dispersal syndrome in the Natural Regeneration Area, RNaut $=$ number of regenerants with autochorous dispersal syndrome in the Natural Regeneration Area, TPane = number of regenerants with anemochoric dispersal syndrome in the mixed native species Tree Planting Area, TPzoo = number of regenerants with zoochoric dispersal syndrome in the mixed native species Tree Planting Area, TPaut = number of regenerants with autochorous dispersal syndrome in the mixed native species Tree Planting Area. Letters above boxes indicates statistically significant differences. 


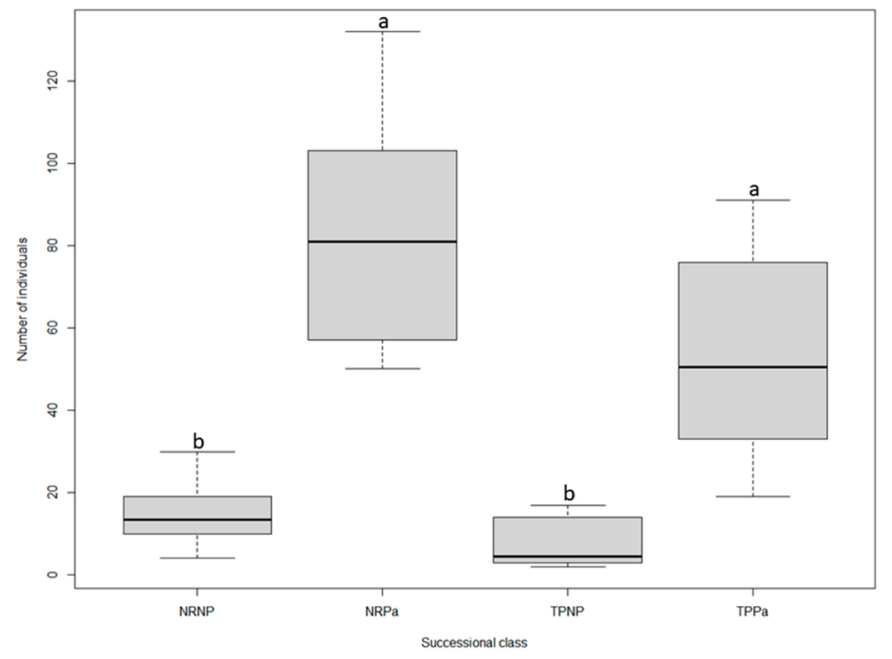

Figure 5. Boxplot of the abundance of regenerants in each successional class at the Borborema region, state of São Paulo, Brazil. Legend: $\mathrm{NRPa}=$ abundance of pioneer regenerants in the Natural Regeneration Area, NRNP $=$ abundance of non-pioneer regenerants in the Natural Regeneration Area, TPPa = abundance of pioneer regenerants in the mixed native species Tree Planting Area and TPNP = abundance of non-pioneer regenerants in the mixed native species Tree Planting Area. Letters above boxes indicates statistically significant differences.

\section{DISCUSSION}

In both restoration methods, the composition, richness, and abundance of families were similar to those found in other areas of natural regeneration and native forests. The families Asteraceae, Malvaceae, Fabaceae, and Solanaceae are frequently found in areas undergoing natural regeneration (Engel \& Parrota, 2001; Alvarenga et al., 2006; Bertocine \& Rodrigues, 2008; Schorn et al., 2009) and in seasonal forests (Garcia et al., 2011; Sciopini et al., 2013; Fernandes et al., 2018). Previous studies have shown that Asteraceae, Malvaceae, and Solanaceae occur in more open areas and forest gaps associated with environments with high luminosity and under natural regeneration (Bertocine \& Rodrigues, 2008; Seubert et al., 2017; Ubert et al., 2018). In the ANR treatment we observed these families among shrubs and pioneer herbaceous species. We observed the same for Fabaceae, which is one the most abundant families in seasonal forests (Garcia et al., 2011; Sciopini et al., 2013; Fernandes et al., 2018).

Most species were trees, shrubs, and herbs in the two restoration methods and most species richness comes from zoochoric and pioneer species, a typical pattern in areas at an early stage of regeneration (Engel \& Parrota, 2001; Bertocine \& Rodrigues, 2008). However, the studied areas showed differences in composition, structure, density, growth forms, and dispersal syndromes. The ANR treatment presented a higher density of individuals than the TP treatment, with almost twice as many regenerant species. However, these regenerants were mostly shrubs. Many authors argue that this group of plants arrive at the early stages of succession, when a high amount of pioneer shrub and tree species is found, which are later replaced by other species of more advanced successional stages (Yarranton \& Morrison, 1974; Baylão Junior et al., 2013; Coutinho et al., 2019). We observed that even with a high number of pioneer species in the area, non-pioneer plants are present in the ANR treatment. We believe that this area continues to follow a trajectory of succession, since the landscape scenario revealed the presence of fragments that favor the input of seed rain, contributing to the succession process (Vieira \& Scariot, 2006; Prach \& Walker, 2019).

In areas restored using the Assisted Natural Regeneration treatment, many species arrive at the place when propagules are brought by biotic or abiotic dispersers (Gorchov et al., 1993; Vieira \& Scariot, 2006; Pinã-Rodrigues \& Aoki, 2014; Dallabrida et al., 2017). We believe that these dispersers were significant both for the density and richness of the ANR regenerants, because there was a similar input of autochoric, anemochoric, and zoochoric species (Figure 3), despite the predominance of anemochoric herbaceous species of the Asteraceae family.

The similarity of the areas was almost $50 \%$ and, probably, one of the factors that affected the composition of species may be the biotic and abiotic dispersal in the ANR treatment, since the surroundings of the area were characterized by pastures and seasonal forest remnants, which may contribute to the flow of propagules (Arenas et al., 2017). In the mixed native species Tree Planting Area, abiotic dispersal may have played a critical role since $S$. polyphylla was very abundant in this area.

The similarity of regenerant species between the restoration sites considering the seedlings planted in the TP area was lower than $10 \%$. This must be related to the fact that the most planted species have not yet started to reproduce. The trees planted in the TP treatment (Additional file 1) were already well established, presenting a canopy, and were contributing to 
the ecological processes of the area. However, they were not yet mature, nor were producing seeds. This may explain the fact that the sampled regenerating species must be related to the arrival of propagules from the surrounding areas, as other authors have already shown in their studies (Wurdele jr, 1997; PinãRodrigues \& Aoki, 2014; Arenas et al., 2017).

\subsection{Phytosociology}

The majority of the tree species with the highest Importance Value Index (IVI) in the ANR treatment came from external communities since they are species different from those planted in the TP treatment. The two species that presented the highest Importance Value Index (IVI), S. mauritianum and S. paniculatum, belong to the Solanaceae family. These species have already been pointed out as pioneer species in forest restoration areas (Ferreira \& Vieira, 2017; Seubert et al., 2017). S. mauritianum occurred in all sampled plots, which contributed to the observed high IVI, the high relative dominance, and the high frequency of the species. On the other hand, regarding S. paniculatum, the high IVI was related to the high density of individuals, with the highest value found in the study area and also due to its relative high frequency. The third most important species in the study area, P. guajava, showed large stems and a high relative dominance, being found in all plots with a high frequency. This species is considered to be a pioneer, presenting a zoochoric dispersal syndrome, and being highly appreciated by birds (Coutinho et al., 2019). Also, M. fistulifera and A. aculeata are among the species with the highest IVI and intermediate values of density, frequency, and relative dominance. However, M. fistulifera, which is autochoric, and one of the species found in areas adjacent to the study, is probably introduced in the area by dispersion.

In the TP treatment, the tree species with the highest Importance Value Index were already planted in 2014, except for S. paniculatum, which was found in the fragment adjacent to the experiment. The high density, frequency, and relative dominance of S. polyphylla contributed to the high IVI of this species, since it had many individuals and regenerants in almost all the plots. S. polyphylla is an autochoric pioneer species, widely used in restoration areas due to its high density populations (Durigan et al., 2016). S. paniculatum was second in the IVI ranking, since it had a high relative dominance in the area, followed by H. ochraceus, A. virgata, and S. terebinthifolia, which presented intermediate values of density, frequency, and dominance.

The restoration areas already have defined regenerating communities. However, they showed differences in floristic composition, life forms, and similarity, and the planted tree species contributed little to the community of regenerant plants, up to four years after planting.

\subsection{Feasibility of restoration methods}

The Tree Planting method is more common than the Assisted Natural Regeneration in restoration, despite the economic and environmental potential of the assisted regeneration (Chazdon \& Gariguata, 2016), since it is widely known that the success of ecological restoration is higher for natural regeneration (Crouzeiles et al. 2017). Nevertheless, it is still important to design case studies to investigate the role of natural regeneration for tropical restoration (Uriarte \& Chazdon, 2016). In our study, the Assisted Natural Regeneration was similar to the assisted Tree Plantation (TP) regarding the floristic composition of regenerants, with a higher number of zoochoric species and individuals, despite the lower abundance of tree species. The restoration trajectory appears to be occurring faster in the ANR treatment. ANR areas presented species that are not commonly produced in forest nurseries, a fact that may increase the richness of restoration projects, providing opportunities for the fauna and for the plant species themselves. Considering the costs of seedling production, transportation, and the maintenance practices carried out in both areas, it is possible to observe that, so far, the ANR technique has been more efficient than the TP treatment in promoting the return of the diversity, structure, and potential attractiveness for the fauna, as well as providing soil cover.

The remnants of natural vegetation surrounding the restoration areas tend to foster the input of seeds and the increase of density of natural regeneration closer to the border of these remnants (Viana et al., 2010). A recent review demonstrated that the landscape configuration drives the recovery of the density and richness of species, depending more on the soil conditions, and especially on the distance and size of the nearest forest remnants than on the richness and density of the planted seedlings (Suganuma et al., 2018). However, the evolution of restoration in seasonal forests depends on the age of the restoration, which is the strongest factor driving most community attributes (Suganuma \& Durigan, 2015). Therefore, based on these studies and on our results, we can propose that, for both areas, the succession process still depend on the landscape matrix and on the time elapsed after the beginning of the restoration.

\section{CONCLUSION}

We concluded that the two restoration methods allowed the regeneration of native plants, forming communities with high numbers of pioneer species. The natural regeneration was a more efficient strategy for the abundance of regenerating individuals and for the variety of dispersion syndromes. The two techniques were able to boost the initial processes of community regeneration in a matrix surrounded by forest remnants. 


\section{ACKNOWLEDGEMENTS}

The authors thank the company AES Brasil, for providing all human and material resources for the field experiment, especially to the Licensing Conditions Coordinator, Veronez, O. ; research assistants from CEIBA Consultoria Ambiental, for assisting in the implementation and maintenance of the field experiment.

\section{SUBMISSION STATUS}

Received: 16 Oct. 2020

Accepted: 29 Apr. 2021

Associate editor: Rodrigo Studart Corrêa (D)

\section{CORRESPONDENCE TO}

\section{Bruno Santos Francisco}

Universidade Federal de Sao Carlos, Rod. João Leme dos Santos km 110, 264, 18052-780, Bairro do Itinga, Sorocaba, SP, Brasil

e-mail: brunofrancisco@estudante.ufscar.br

\section{SUPPLEMENTARY MATERIAL}

The following online material is available for this article: Additional file 1. List of species planted in 2014 in mixed native tree planting (TP) in the region of Borborema, São Paulo State, Brazil.

\section{REFERENCES}

Almeida DS. Recuperação ambiental da Mata Atlântica. $3^{\text {a }}$ edn. Ilhéus: Editus; 2016.

Alvarenga AP, Botelho AS, Pereira IM. Avaliação da regeneração natural na recomposição de matas ciliares em nascentes na região sul de Minas Gerais. Cerne 2006; 12 (4): 360-372.

Arenas, JM, Lázaro-Lobo A, Mola I, Escudero A, Casado MA. The influence of site factors and proximity of adjacent vegetation on tree regeneration into roadslopes. Ecological Engineering 2017; 101: 120-129.

Baylão junior HFB, Valcarcel R, Nettesheim FC. Fatores do meio físico associado ao estabelecimento de espécies rústicas em ecossistemas perturbados na Mata Atlântica, Piraí, RJ-Brasil. Ciência Florestal 2013; 3 (3): 305-315.

Barbosa LM, Shirasuna RT, Lima FD, Ortiz PRT. Lista de espécies indicadas para restauração ecológica para diversas regiões do estado de São Paulo. São Paulo, Instituto de Botânica; 2017.

Bastin JF, Finegold Y, Garcia C, Mollicone D, Rezende M, Routh D, et al. The global tree restoration potential. Science 2019; 365 (6448): 76-79.

Brasil. Decreto no 8.972, de 23 de janeiro de 2017. Diário Oficial da República Federativa do Brasil, Brasília, DF.

Bertoncini AP, Rodrigues RR. Forest restoration in an indigenous land considering a forest remnant influence (Avaí, São Paulo State, Brazil). Forest Ecology and Management 2008; 255 (3-4): 513-521.
Calegari L, Martins SV, Campos LC, Silva E, Gleriani JM. Avaliação do banco de sementes do solo para fins de restauração florestal em Carandaí, MG. Revista Árvore 2013; 37 (5): 871-880.

Campello EFC. Sucessão vegetal na recuperação de áreas degradadas. In Dias LE, Mello JWV, editores. Recuperação de áreas degradadas. Viçosa: UFV; Departamento de Solos; Sociedade Brasileira de Recuperação de Áreas Degradadas; 1998.

Chazdon RL, Guariguata MR. Natural regeneration as a tool for large-scale forest restoration in the tropics: prospects and challenges. Biotropica 2016; 48 (6): 716-730.

Chazdon RL, Bodin B, Guarig-uata M, Lamb D, Walder B, Chokkalingam $U$, et al. Partnering with nature: the case for natural regeneration in forest and landscape restoration. FERI Policy Brief; Montreal 2017.

Climate-data.org. Dados meteorológicos do projeto OpenStreetMap, coletados entre 1982 e 2012. [cited Oct.13, 2020]. Available at https://pt.climate-data.org/america-do-sul/brasil/sao-paulo/ borborema-34940/\#climate-graph.

Coutinho PRDO, Valcarcel R, Rodrigues PJFP, Braga JMA. Restauração passiva em pastagens abandonadas a partir de núcleos de vegetação na Mata Atlântica, Brasil. Ciência Florestal 2019; 29 (3): 13

Crouzeilles R, Ferreira MS, Chazdon RL, Lindenmayer DB, Sansevero JB, Monteiro L, et al. Ecological restoration success is higher for natural regeneration than for active restoration in tropical forests. Science Advances 2017; 3 (11): e1701345. 07-1323.

Dallabrida JP, Silva ACD, Higuchi P, Souza K, Loebens R, Rodrigues Júnior LC, et al. Elementos nucleadores da paisagem influenciam a dispersão zoocórica em áreas campestres alto-montanas. Rodriguesia 2017; 68 (2): 325-335.

Durigan G, Suganuma MS, Melo, ACG. Expected values for attributes of riparian forests undergoing restoration at different ages. Scientia Forestalis 2016; 44 (110): 463-474.

Engel VL, Parrotta JA. An evaluation of direct seeding for reforestation of degraded lands in central Sao Paulo state, Brazil. Forest Ecology and Management 2001; 152 (1-3): 169-181.

Fernandes MM, Andrade GKO, Ferreira RA, Silva TR, Souza IBA, Magalhães JS. Regeneração natural em área de reflorestamento misto com espécies nativas no município de Laranjeiras, SE. Revista de Ciências Agrárias Amazonian Journal of Agricultural and Environmental Sciences 2018; 61: 1-9.

Ferreira, MC, Vieira DLM. Topsoil for restoration: Resprouting of root fragments and germination of pioneers trigger tropical dry forest regeneration. Ecological Engineering 2017; 103: 1-12.

Freitas MG, Rodrigues SB, Campos-Filho EM, do Carmo, GHP, da Veiga JM, Junqueira RGP, et al. Evaluating the success of direct seeding for tropical forest restoration over ten years. Forest ecology and management 2019; 438: 224-232.

Garcia CC, Reis MDGF, Reis GGD, Pezzopane JEM, Lopes HNS, Ramos DC. Regeneração natural de espécies arbóreas em fragmento de Floresta Estacional Semidecidual Montana, no domínio da Mata Atlântica, em Viçosa, MG. Ciência Florestal 2011; 21 (4): 677-688.

Gorchov DL, Cornejo F, Ascorra C, Jaramillo M. The role of seed dispersal in the natural regeneration of rain forest after strip-cutting in the Peruvian Amazon. Vegetatio 1993; 107 (1): 339-349. 
Koeppen, W. Climatología: con un estudio de los climas de la Tierra. Mexico City: Fondo de Cultura Economica; 1948.

Leal-filho N, Santos GR, Ferreira RL. Comparando técnicas de nucleação utilizadas na restauração de áreas degradadas na Amazônia brasileira. Revista Árvore 2013; 37 (4): 587-597.

Martins FR, Santos FAM. 1999. Técnicas usuais de estimativa da biodiversidade. Revista Holos 1 (1): 236-267.

Moro MF, Martins FR. Métodos de levantamento do componente arbóreo-arbustivo. In: Felfili JM, Eisenlohr PV, Melo MMRF, Andrade LA, Meira Neto JAA, Editors. Fitossociologia no Brasil: métodos e estudos de caso. Viçosa: Editora UFV; 2011.

Oliveira RE, Engel VL. Indicadores de monitoramento da restauração na Floresta Atlântica e atributos para ecossistemas restaurados. Scientia Plena 2018; 13 (12): 1-13.

Passaretti RA, Pilon NAL, Durigan G. Weed control, large seeds and deep roots: drivers of success in direct seeding for savanna restoration. Applied Vegetation Science 2020; 23: 406-416.

Piña-rodrigues FCM, Aoki J. Chuva de sementes como indicadora do estádio de conservação de fragmentos florestais em Sorocaba - SP. Ciência Florestal 2014; 24 (4): 911-923.

Planaveg. Portaria Interministerial no 230, de 14 de novembro de 2017. PLANAVEG (Plano Nacional de Recuperação da Vegetação Nativa), Ministério do Meio Ambiente, Ministério da Agricultura, Pecuária e Abastecimento, Ministério da Educação (Aug. 24, 2020).

Prach, K, Walker, LR. Differences between primary and secondary plant succession among biomes of the world. Journal of Ecology 2019; 107 (2): 510-516.

R Core Team. R: A language and environment for statistical computing. R Foundation for Statistical Computing, Vienna, Austria. [cited Nov 15, 2020]. Available at http://www.R-project.org/.

Rodrigues SB, Freitas MG, Campos-Filho EM, do Carmo GHP, da Veiga JM, Junqueira RGP, et al. Direct seeded and colonizing species guarantee successful early restoration of South Amazon forests. Forest Ecology and Management 2019; 451: 117559.

Reis DN, Davide AC, Ferreira DF. Indicadores preliminares para avaliação da restauração em reflorestamentos de ambientes ciliares. Pesquisa florestal brasileira 2014; 34 (80): 375-389.

Santos PS. Influência do entorno sobre a chuva de sementes e deposição de serapilheira em fragmentos de floresta atlântica. [Ph.D. Thesis]. Recife: Universidade Federal Rural de Pernambuco; 2018.
Schorn LA, Krieger A, Nadolny MC, Fenilli TABLE Avaliação de técnicas para indução da regeneração natural em área de preservação permanente sob uso anterior do solo com Pinus elliottii. Floresta 2010; 40 (2): 1-14.

Scipioni MC, Finger CAG, Cantarelli EB, Denardi L, Meyer EA. Fitossociologia em fragmento florestal no noroeste do estado do Rio Grande do Sul. Ciência Florestal 2011; 21 (3): 409-419.

Secretaria de assuntos estratégicos (SAE). Impacto da revisão do código florestal: como viabilizar o grande desafio adiante?. [cited 2020 nov. 15]. Available at http://www.sae.gov.br/site/wp-content/uploads/.

Seubert RC, Macaneiro JP, Schorn LA, Sebold DC. Natural regeneration in different periods of abandonment of areas after the extraction of Eucalyptus grandis Hill ex Maiden in red-yellow ultisol álic, in Brusque, Santa Catarina state. Ciência Florestal 2017; 27 (1): 1-19.

Soares-filho B, Rajão R, Macedo M, et al. 2014. Cracking Brazil's Forest Code. Science 344: 363-364.

Soares-Filho B, Rajão R, Macedo M, Carneiro A, Costa W, Coe M, et al. Cracking Brazil's forest code. Science 2014; 344 (6182): 363-364.

Souza LM, Faria RAVB, Botelho AS, Fontes MAL, Faria JMR. Potencial da regeneração natural como método de restauração do entorno de nascente perturbada. Cerne 2012; 18 (4): 565-576.

The plant list. Version 1.1. [cited 2020 nov. 15]. Available at http://www.theplantlist.org/.

Uberti GM, Schulte TF, Farias JA, Welter, CA, Silva RR. Avaliação da regeneração/rebrota em uma clareira que sofrera exploração ilegal de madeira nativa no município de Segredo, Rio Grande do Sul. Caderno de Pesquisa 2018; 30 (3): 1-12.

Uriarte $\mathrm{M}$, Chazdon RL. Incorporating natural regeneration in forest landscape restoration in tropical regions: synthesis and key research gaps. Biotropica 2016; 48 (6): 915-924.

Vieira DLM, Scariot A. Principles of natural regeneration of tropical dry forests for restoration. Restoration ecology 2006; 14 (1): 11-20.

Yarranton GA, Morrison RG. Spatial dynamics of a primary succession: nucleation. The Journal of Ecology 1974; 62: 417-428.

Wunderle Jr, Joseph M. The role of animal seed dispersal in accelerating native forest regeneration on degraded tropical lands. Forest Ecology and Management 1997; 99 (1-2): 223-235. 